\title{
Effect of Passive Movements of Lower Extremity on Hemodynamic Parameters of the Patients under Ventilator
}

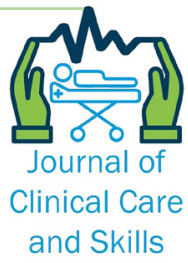

\section{ARTICLE INFO}

\section{Article Type}

Original Research

\section{Authors}

Rezaeikia R. ${ }^{1} M S c$

Najafi Dolatabad Sh.* MSc Afrasiabifar A. ${ }^{2} P h D$,

Zoladl M. ${ }^{3} P h D$

How to cite this article
Rezaeikia R, Najafi Dolatabad Sh,
Afrasiabifar A, Zoladl M. Effect of
Passive Movements of Lower Ext-
remity on Hemodynamic Param-
eters of the Patients under Vent-
ilator. Journal of Clinical Care and
Skills. 2020;1(1):37-42.

*Medical Surgical Nursing Department, Yasuj University of Medical Sciences, Yasuj, Iran

${ }^{1}$ Student Research Committee, Yasuj University of Medical Sciences, Yasuj, Iran

${ }^{2}$ Medical Surgical Nursing Department, Yasuj University of Medical Sciences, Yasuj, Iran

${ }^{3}$ Social Determinants of Health Research Center, Yasuj University of Medical Sciences, Yasuj, Iran

\section{Correspondence}

Address: Medical Surgical Nursing Department, Nursing \& Midwifery Faculty, Yasuj University of Medical Sciences, Dr. Jalil Street, Yasuj, Iran

Phone: +98 (74) 33235141

Fax: +98 (74) 33235141

najafi.shahla@yums.ac.ir

\section{Article History}

Received: November 1, 2017

Accepted: January 20, 2018

ePublished: March 10, 2020

\section{A B S T R A C T}

Aims The present study was conducted with the aim of determining the effect of passive movements on hemodynamic parameters, including systolic blood pressure, diastolic blood pressure, mean arterial pressure, pulse, and pulse pressure of patients under ventilation. Materials \& Methods In this controlled clinical trial, 64 patients under ventilation in Kohgiluyeh and Boyer-Ahmad University of Medical Sciences and Health Services and Shiraz University of Medical Sciences were selected by convenience sampling method. They were assigned to experimental (32 people) and control (32 people) groups, using random block assignment. The experimental group, in half supine position at $30^{\circ}$, received passive movements of the lower extremities, according to the protocol, by a therapist for 22 minutes. Then, the values of hemodynamic parameters were recorded after 22 minutes and at intervals of 2, 6, 12 and $24 \mathrm{~h}$ after the post-test, as follow-up times. Data were analyzed by SPSS 21 software, using independent t-test, Chi-square test and repeated measure of variance analysis.

Findings In hemodynamic parameters of the experimental group in each time interval, including immediately, 2 hours, 6 hours, 12 hours, and 24 hours after the completion of the research interventions, compared with their amount at the starting point of the study, and in comparison with these changes in the control group, there was no significant difference $(\mathrm{p}>0.05)$.

Conclusion Passive movements of lower extremity have no effect on hemodynamic parameters, including systolic blood pressure, diastolic blood pressure, mean arterial pressure, pulse, and pulse pressure of patients under ventilation.

Keywords Passive movements; Lower extremity; Hemodynamic; Ventilator

\section{CIT A T I O N L I N KS}

[1] Harrison's Principles of Internal ... [2] Evaluation of need for mechanical ventilation in children who hospitalized ... [3] Intensive care unit; Glimmer of hope for patients with acute ... [4] A comparative study between S.I.M.V to T-piece and ... [5] Study on value of Arterial blood gas measurement ... [6] Efficacy and safety of a paired sedation and ventilator weaning protocol ... [7] Reversibility of lung collapse and hypoxemia in early acute respiratory ... [8] Merriam-webster's medical ... [9] Non invasive hemodynamic monitoring in critical care ... [10] Recommendations on basic requirements for ... [11] Principles and practice of mechanical ... [12] Preventing complications of central venous ... [13] A multiple-center survey on the use in clinical practice of noninvasive ventilation ... [14] The effect of bed rest and potential of prehabilitation on patients in the ... [15] The immediate effects of deep breathing exercises on atelectasis and oxygenation ... [16] What are the hemodynamic and respiratory effects ... [17] Effects of positioning and exercise on intracranial pressure in a ... [18] The Cardiopulmonary effect of passive ... [19] Influence of passive leg movements on blood circulation on the tilt table in ... [20] The effect of extremity range of motion ... [21] The effects of therapeutic passive movement on ... [22] Physiological responses to passive exercise ... [23] Passive leg ... [24] Passive leg movement-induced hyperaemia with ... [25] Hemodynamic and metabolic effects of passive ... [26] Central and peripheral contributors to skeletal muscle ... [27] "Passive exercise" using whole body periodic ... [28] Blood flow velocity and vascular resistance ... [29] Effectiveness of passive range of motion exercise on hemodynamic parameters and behavioral ... [30] Effect of passive range of motion on ... [31] Very early passive cycling exercise in mechanically ventilated critically ill patients: Physiological and safety ... [32] Marino's The ICU ... [33] Comprehensive book on intensive nursing care in ICU,CCU and dialysis ... 


\section{Introduction}

One of the most vital systems of the body is the respiratory system; since any changes in the function of this system cause interruptions in respiratory gas exchange and inadequate pulmonary ventilation, doing necessary actions to meet the requirements of ventilation and improving oxygenation to tissues are the most important medical requirements [1,2]. These interventions are more important in patients hospitalized in ICU; since these patients are very ill because of severe disorders in vital systems of the body, and more than $75 \%$ of them require mechanical ventilation through artificial airway and ventilator connection due to severe respiratory problems [3,4], through which it maintains an adequate level of arterial oxygen pressure and arterial carbon dioxide pressure and prevents additional respiratory work by providing artificial support for gaseous exchange and respiration, and until the elimination of the cause of respiratory dysfunction and the return of life threatening conditions including hypoxemia and acidosis progressive respiration, respiratory muscle fatigue may be stopped and necessary support for the lungs may be provided [5].

Despite the wide usage range of ventilator, the results of studies have shown that this device can improve the patient's respiratory state, but reduce the health level of the patient by making negative changes in hemodynamic parameters as well as causing other side effects [6,7]. As the principles of dynamic blood flow, hemodynamic is a part of the knowledge of physiology or medical science [8], and its monitoring traditionally provides useful information on cardiac output and fluid replacement, such as systemic blood pressure, heart rate, central venous pressure, and cardiac output. It is experiencing constant development with the advancement of new technologies and the addition of new indices. Today, hemodynamic examination in patients with severe illness accounts for a large part of the care of these patients [9].

Nowadays, proper interventions to prevent and control the direct and indirect negative effects of mechanical ventilation on hemodynamic parameters are among the tasks of nurses. Although negative effects on hemodynamic parameters such as hypotension, arterial pressure drop, heart rate change, reversible inferiority, preload decrease, and cardiac output decrease are the direct negative effects of mechanical ventilation on hemodynamic parameters, it should be noted that in patients under the ventilator, the indirect negative effects of mechanical ventilation on hemodynamic parameters are significant due to some reasons, including immobility, muscle mass loss, bed sores, edema, systemic dependence, gastrointestinal ulceration, increased intracranial pressure, intravascular coagulation, ankle dislocation, and external displacement of the thigh and leg joints [10,11].

Journal of Clinical Care and Skills
Although the use of drug methods has been common to prevent complications since now, as they are expensive and costly on one hand, and the normal maintenance of hemodynamic parameters in patients under ventilation is strongly recommended on the other hand, attention to other treatments will be of particular importance in this regard [12,13].

Lack of movement is one of the predisposing or exacerbating causes of most of the mentioned complications. One of the simplest and least costly interventions to deal with these complications is physical activity that speeds up blood flow and improves the functioning of the heart; doing exercise and having physical activities, patients can improve their functional status, increase their quality of life, facilitate their separation from the ventilator, and reduce hospital stay $[14,15]$. Thus, actions and interventions such as passive movements of the lower extremities that are performed solely by a therapist, in which the patients do not play a role, can be used in this regard [16-18].

While some studies suggest that these movements can affect blood flow, prevent syncope [19], prevent dryness and stiffness of the joints, vascular clots, muscle cramps and muscle tone reduction, and improve the hemodynamic parameters of patients under ventilation [15], but the results of some studies indicate that the inactive movement of the lower extremity is not affected in this regard [20-22].

Regarding the inconsistent and results of the studies and the need for effective and necessary interventions to prevent, control, or improve the hemodynamic symptoms in patients under ventilation by nurses, as the most important member of the care and therapeutic team, the present study was conducted with the aim of determining the effect of passive movements on hemodynamic parameters, including systolic blood pressure, diastolic blood pressure, mean arterial pressure, pulse, and pulse pressure of patients under ventilation.

\section{Materials and Methods}

The present controlled clinical trial was conducted in a community of patients under ventilation in Kohgiluyeh and Boyer-Ahmad University of Medical Sciences and Health Services and Shiraz University of Medical Sciences. By referring to the intensive care units affiliated to Kohgiluyeh and Boyer-Ahmad University of Medical Sciences and Shiraz University of Medical Sciences, 64 patients were selected by convenience sampling method; after contacting their legal parents and explaining the aims of the study and obtaining their informed consent, they were assigned to experimental (32 people) and control (32 people) groups, using random block assignment. The inclusion criteria in this study included no congestive heart failure, acute myocardial infarction, thrombocytopenia, and active rheumatoid arthritis; no fracture, amputation, congenital anomalies, and 
deep vein thrombosis in the lower extremities and no damage in the spinal cord.

After classifying the research subjects to the groups, the demographic information, including age, gender, body mass index, primary diagnosis, the consciousness level in Glasco and mechanical venting were collected for all of them. Their hemodynamic parameters, including systolic blood pressure, diastolic blood pressure, mean arterial pressure, pulse rate, and pulse pressure were measured and recorded by the vital signs monitoring (Saadat S740; Iran).

In this research, all ethical research points such as obtaining the informed written consent of the legal parents of the research subjects, assuring them of being allowed to leave the study at any time they would not continue, respecting the confidentiality of the data collection, and stop changing the drugs or depriving the research subjects from standard treatment were observed; the present study was approved by the research ethics committee (IR.YUMS.REC.1395.4) at Iranian clinical trial site with IRCT2016041727444N1 code. In the control group, individuals received only routine care and treatment. The participants of the experimental group, in half supine position at $30^{\circ}$, received passive movements of the lower extremities, according to the protocol, by a therapist for 22 minutes (Table 1) [17, 23-26].

Table 1) Protocol of passive lower limbs for the experimental group during 22 minutes

\begin{tabular}{|c|c|}
\hline Movement & Intervention description \\
\hline 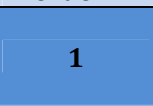 & $\begin{array}{l}\text { Raise the right leg of the patient directly at } 45^{\circ} \\
\text { and keep it in this position for one minute and } \\
\text { perform the same for the left leg. }\end{array}$ \\
\hline 2 & $\begin{array}{l}\text { Up and down right ankle at } 20^{\circ} \text { to } 50^{\circ}, 30 \text { times } \\
\text { per minute for one minute and similar on the } \\
\text { left ankle. }\end{array}$ \\
\hline 3 & $\begin{array}{l}\text { The right ankle rotation movements } 30 \text { times } \\
\text { per minute in clockwise direction for one } \\
\text { minute and the same for the left ankle. }\end{array}$ \\
\hline 4 & $\begin{array}{l}\text { The right ankle rotation movements } 30 \text { times } \\
\text { per minute in counterclockwise direction for } \\
\text { one minute and the same for the left ankle. }\end{array}$ \\
\hline 5 & $\begin{array}{l}\text { Raise the patient's right leg directly at } 45^{\circ} \text { and } \\
\text { keep it in this position for one minute and } \\
\text { perform the same on the left leg. }\end{array}$ \\
\hline 6 & $\begin{array}{l}\text { Bend and straight the right knee joint at a } 90^{\circ} \text {, } \\
30 \text { times per minute for two minutes and } \\
\text { perform the same on the left leg. }\end{array}$ \\
\hline 7 & $\begin{array}{l}\text { Raise the right leg of the patient directly at } 45^{\circ} \\
\text { and keep it in this position for one minute and } \\
\text { perform the same for the left leg. }\end{array}$ \\
\hline 8 & $\begin{array}{l}\text { Open and close the right hip at a } 90^{\circ}, 30 \text { times } \\
\text { per minute for two minutes and perform the } \\
\text { same for the left hip joint. }\end{array}$ \\
\hline 9 & $\begin{array}{l}\text { Raise the right foot of the patient directly at } 45 \\
\text { 'and keep it in this position for one minute and } \\
\text { perform the same for the left leg. }\end{array}$ \\
\hline
\end{tabular}

After 22 minutes, in order to prevent bias and keep the blind nature of the research, a person who did not know the assignment of the research participants to the experimental or control group, observed the hemodynamic parameters of all the patients from the data collection tools screen and recorded them as a post-test. Then, the values of these parameters were recorded at intervals of 2 hours, 6 hours, 12 hours and 1 day after the posttest, as follow-up times.

Data analysis was performed, using SPSS 21 software, by a statistician who was blind in relation to the code assigned to each of the groups. To examine the normal distribution of data, the Kolmogorov-Smirnov test was used; to compare the intergroup hemodynamic parameters at the starting point of the study, independent t-test and Chi-square test were used; to compare the intergroup qualitative variables (gender, primary diagnosis and mechanical ventilation method with a ventilator) and to compare the intergroup hemodynamic parameters of patients under ventilator in terms of time/group, a repeated measure of variance analysis was used.

\section{Findings}

A total of 64 patients hospitalized under ventilation, with a mean age of $59.1 \pm 20.8$ years, BMI of $25.7 \pm 5.9 \mathrm{Kg} / \mathrm{m}^{2}$, and a mean level of consciousness of 7.2 \pm 2.7 participated in this study (Table 2).

There was no significant difference between the groups in terms of age, body mass index, and consciousness, as well as qualitative variables, including gender, primary diagnosis, and mechanical ventilation method at the starting point of the study. Also, in hemodynamic parameters of the experimental group in each time interval including immediately, 2 hours, 6 hours, 12 hours, and 24 hours after the completion of the research interventions, compared with their amount at the starting point of the study, and in comparison with these changes in the control group, there was no significant difference.

There was no significant difference between the mean and standard deviation of hemodynamic parameters between the 2 groups (Table 3 ).

Table 2) Absolute frequency and frequency of gender, primary diagnosis, and mechanical ventilation with ventilator devices in

\begin{tabular}{|c|c|c|}
\hline Variables & Number & Percentage \\
\hline \multicolumn{3}{|l|}{ Gender } \\
\hline Man & 39 & 60.9 \\
\hline Woman & 25 & 39.1 \\
\hline \multicolumn{3}{|l|}{ Primary diagnosis } \\
\hline Respiratory disorders & 16 & 25.0 \\
\hline Nervous system disorders & 15 & 23.4 \\
\hline Liver disorders & 5 & 7.8 \\
\hline Renal disorders & 9 & 14.1 \\
\hline Complications after surgery & 6 & 9.4 \\
\hline Others & 13 & 20.3 \\
\hline \multicolumn{3}{|c|}{ Mechanical ventilation method } \\
\hline SIMV & 42 & 65.6 \\
\hline BIPAP & 7 & 10.9 \\
\hline CPAP & 6 & 9.4 \\
\hline SPONT & 6 & 9.4 \\
\hline Others & 3 & 4.7 \\
\hline
\end{tabular}


Table 3) Intergroup comparison between mean and standard deviation of hemodynamic parameters of patients under ventilator in terms of time/group

\begin{tabular}{|c|c|c|c|c|c|c|c|}
\hline $\begin{array}{l}\text { Hemodynamic } \\
\text { parameters }\end{array}$ & $\begin{array}{c}\text { Starting point } \\
\text { of study }\end{array}$ & $\begin{array}{l}\text { Immediately after } \\
\text { the intervention }\end{array}$ & $2 h$ after & $6 h$ after & $12 \mathrm{~h}$ after & $24 \mathrm{~h}$ after & P. Value \\
\hline \multicolumn{8}{|l|}{ Systolic blood pressure } \\
\hline Experimental group & $26.2 \pm 129.6$ & $25.9 \pm 126.1$ & $24.7 \pm 124.3$ & $26.4 \pm 127.3$ & $30.1 \pm 130.5$ & $25.2 \pm 135.1$ & \multirow{2}{*}{0.09} \\
\hline Control group & $20.1 \pm 123.3$ & $24.6 \pm 122.6$ & $21.4 \pm 121.8$ & $17.5 \pm 125.2$ & $20.0 \pm 122.1$ & $22.7 \pm 117.7$ & \\
\hline \multicolumn{8}{|l|}{ Mean diastolic pressure } \\
\hline Experimental group & $16.9 \pm 80.3$ & $18.5 \pm 79.3$ & $15.0 \pm 75.7$ & $19.4 \pm 77.5$ & $20.6 \pm 78.9$ & $18.7 \pm 82.8$ & \multirow{2}{*}{0.23} \\
\hline Control group & $17.4 \pm 76.8$ & $18.5 \pm 74.8$ & $16.7 \pm 74.9$ & $14.0 \pm 75.8$ & $16.5 \pm 74.7$ & $17.5 \pm 72.3$ & \\
\hline \multicolumn{8}{|l|}{ Mean arterial pressure } \\
\hline Experimental group & $18.9 \pm 96.7$ & $20.5 \pm 94.9$ & $17.3 \pm 91.9$ & $21.3 \pm 94.1$ & $22.4 \pm 96.1$ & $20.0 \pm 100.2$ & \multirow{2}{*}{0.16} \\
\hline Control group & $17.1 \pm 92.3$ & $19.3 \pm 90.7$ & $17.0 \pm 90.6$ & $13.1 \pm 92.2$ & $15.8 \pm 90.5$ & $18.4 \pm 87.4$ & \\
\hline \multicolumn{8}{|l|}{ Pulse rate } \\
\hline Experimental group & $20.1 \pm 93.3$ & $20.0 \pm 97.1$ & $20.9 \pm 93.5$ & $23.7 \pm 940.8$ & $24.1 \pm 94.5$ & $19.9 \pm 91.2$ & \multirow{2}{*}{0.08} \\
\hline Control group & $17.8 \pm 96.7$ & $18.0 \pm 94.6$ & $19.0 \pm 95.4$ & $18.3 \pm 91.3$ & $16.3 \pm 91.0$ & $18.3 \pm 91.4$ & \\
\hline \multicolumn{8}{|l|}{ Pulse pressure } \\
\hline Experimental group & $16.9 \pm 49.3$ & $12.1 \pm 46.7$ & $15.8 \pm 48.6$ & $11.7 \pm 49.8$ & $19.5 \pm 51.6$ & $14.5 \pm 52.3$ & \multirow{2}{*}{0.32} \\
\hline Control group & $14.3 \pm 46.5$ & $16.0 \pm 47.8$ & $15.2 \pm 46.9$ & $16.6 \pm 49.4$ & $17.2 \pm 47.4$ & $13.1 \pm 45.4$ & \\
\hline
\end{tabular}

\section{Discussion}

The aim of this study was to evaluate the effect of passive movements of lower extremity on hemodynamic parameters (systolic blood pressure, diastolic blood pressure, mean arterial pressure, pulse, and pulse pressure) of patients under ventilation.

The results of studies conducted by Brimioulle et al., Achugbue, Fukuda, et al., and Thelandersson et al. have supported the findings of the present study, stating that no change in systolic blood pressure occurred by passive movement $[17,21,27,28]$. Unlike the above consistent studies, Younis and Sayed Ahmed did not support the results of this study; in their study, after passive movement, as a research intervention, systolic blood pressure has significantly decreased [29]. Explaining this difference, it can be noted that passive movement was performed on the whole body in the study, but the independent variable of the present study was to perform passive movements of the lower extremity. The findings of the studies carried out by Brimioulle et al. and Thelandersson et al. have supported the findings of the present study that passive movement did not change the diastolic blood pressure [17, 28]. Contrary to the these studies, the findings of the studies conducted by Achugbue and Younis and Sayed Ahmed have not supported the results of this study; after passive movement, as a research intervention, diastolic blood pressure has significantly decreased [21, 29]. The difference in the results of these studies with the findings of the present study could be justified by the fact that in the present study, the mean diastolic blood pressure was in the normal range, while in the study conducted by Achugbue, the subjects of the study were patients with stroke, being half-body paralyzed, and having hypertension, and reduction in diastolic blood pressure changes was more significant in these patients than in the normal control group. It is worth noting that the difference between the results of this study and the findings of the study conducted by Younis and Sayed Ahmed may be due to the fact that passive movement was performed on the whole body in their study, but the independent variable of the present study was to perform passive movements of the lower extremity.

In the context of mean arterial pressure, the most related findings with this study were the results of a study by Savi et al., suggesting that although the passive movements of the lower extremity on patients under ventilation increased arterial pressure, these changes were not statistically significant [25]. Also, there was no statistical significant difference in the mean arterial pressure variation between the experimental and control groups, being in line with the research studies performed by of Genc et al., Amidei and Sole, Koch et al., and Pires-Neto et al. [16, 22, 30,31].

Although the results of the present study were consistent with the findings of the studies, the findings of the Achugbue's study did not look similar to the results of the present study, suggesting that the mean arterial pressure was reduced by passive movement [21]. Since, based on theoretical knowledge, mean arterial pressure was obtained from systolic pressure and diastolic pressure [32, 33], and based on this, it is affected by changes in blood pressure, and in a study conducted by Achugbue, the study subjects were half paralyzed patients with stroke and hypertension, so changes in mean arterial pressure were more significant in these patients than in normal control group.

In the case of pulse rate, the most similar finding with the present study was the results of a study carried out by Savi et al., suggesting that although the passive movements of the lower extremity on the patients under ventilation increased the number of pulses, these changes were not statistically and significantly different ${ }^{[25]}$. The findings of research 
studies by Genc et al., Loram and de Charmoy, Achugbue, Amidei, and Fukuda et al., Thelandersson et al., Koch et al., and Pires-Neto et al., supported the results of the present study, suggesting that there was no significant statistical change in the dependent variable of pulse rate under the influence of passive movements of the lower extremities, as an independent variable of study [16, 18, 21, 22, 27, 28, 30, 31].

Contrary to the above consistent studies, the findings of the studies performed by Brimioulle et al, and Younis and Sayed Ahmed did not support the results of this study; because in the research, after the passive movement, as a research intervention, pulse has significantly increased [17, 29]. This difference may be justified by the fact that in contrast to the present study, whose independent variable was the passive movement of the lower extremity, in the mentioned studies, passive movement was performed on the lower and upper extremities, and a significant increase in pulse after intervention may be due to coexistence of movements on the lower and upper extremities.

Findings of the present study, stating no statistically significant difference in pulse pressure changes due to passive movement of the lower extremity regarding to the lack of significant increase in systolic blood pressure and diastolic blood pressure, as explained before, were scientifically substantiated; Because, based on theoretical knowledge, pulse pressure results in subtraction of systolic pressure from diastolic pressure and, accordingly, is affected by systolic and diastolic pressure [32, 33].

In addition, the findings of a study by Achugbue were similar to the results of the present study, suggesting that pulse pressure variations were not significantly different from passive movement [21]. It is worth noting that according to previous studies, it seems that in this study, much attention have not been paid to hemodynamic parameters.

However, pulse pressure is a blunt volume and can be used as a non-invasive indicator for evaluating the cardiac output of patients under ventilation [33].

Accordingly, it can be claimed that attention to this index was among the positive points of the present study.

It is suggested that in future studies, more extensive research and more precise and sensitive studies be conducted in order to increase the power of differentiation between values for systolic blood pressure, diastolic blood pressure, mean arterial pressure, pulse, and pulse pressure, so that differences can be distinguished statistically significant.

\section{Conclusion}

Passive movements of lower extremity have no significant effect on hemodynamic parameters, including systolic blood pressure, diastolic blood pressure, mean arterial pressure, pulse, and pulse pressure of patients under ventilation.

Acknowledgments: The authors would like to thank the staff of the Faculty of Nursing and Midwifery and Vice-Chancellor of Research and Technology of Yasuj University of Medical Sciences, nurses of the intensive care unit of the hospitals of Kohgiluyeh and Boyerahmad University of Medical Sciences and Shiraz University of Medical Sciences, research subjects, including patients under ventilation hospitalized in the intensive care unit and their families, and all those who contributed in this study.

Ethical Permission: In this research, all ethical research points such as obtaining the informed written consent of the legal parents of the research subjects, assuring them of being allowed to leave their study at any time they would not continue to investigate, respecting the confidentiality of the data collection, and stop changing the consuming drugs or depriving the research subjects from standard treatment were observed; the present study was approved by the research ethics committee (IR.YUMS.REC.1395.4) at the Iranian clinical trial site with IRCT2016041727444N1 code.

Conflicts of Interests: The authors declared no conflict of interests.

Authors' Contribution: Rezaeikia R. (First author), Introduction author/Original researcher (25\%); Najafi Doulatabad Sh. (Second author), Introduction author/Original researcher/Discussion author (25\%); Afrasiabifar A. (Third author), Introduction author/Methodologist (25\%); Zoladl M. (Fourth author), Introduction author/Statistical analyst/Discussion author (25\%)

Funding/Support: This research is part of the master's thesis of the Faculty of Nursing and Midwifery of Yasuj University of Medical Sciences, sponsored by this university.

\section{References}

1- Kasper DL, Fauci AS, Hauser S, Longo D, Jameson JL, Loscalzo J. Harrison's Principles of Internal Medicine. 19th edition. New York: McGraw-Hill Education; 2015.

2- Nakhjavani N. Evaluation of need for mechanical ventilation in children who hospitalized in PICU. J Babol Univ Med Sci. 2005;7(3):79-84. [Persian]

3- Behbahani S, KarimiMaridani M. Intensive care unit; glimmer of hope for patients with acute condition. Med Lab Eng Mag. 2013;13(149):41-4. [Persian]

4- Zahmatkesh MM, Adeli SH, ValizadeHasanloei MA, Niakan-Lahidji M, Moradi-Moghadam O, Farazmand A, et al. A comparative study between S.I.M.V to T-piece and S.I.M.V to spontaneous breathing then T-piece in process of weaning of the patients from mechanical ventilator in intensive care unit. J Urmia Univ Med Sci. 2011;22(4):3228. [Persian]

5- Zojaji kohan MR, Ahasan B, Naseri K. Study on value of Arterial blood gas measurement in therapeutic process of patients with multiple trauma who were depend to 

mechanical ventilator with clinical stability. J Kurdistan Univ Med Sci. 2005;10(2):55-60. [Persian]

6- Girard TD, Kress JP, Fuchs BD, Thomason JW, Schweickert WD, Pun BT, et al. Efficacy and safety of a paired sedation and ventilator weaning protocol for mechanically ventilated patients in intensive care (awakening and breathing controlled trial): A randomised controlled trial. Lancet. 2008;371(9607):126-34.

7- Borges JB, Okamoto VN, Matos GF, Caramez MP, Arantes PR, Barros F, et al. Reversibility of lung collapse and hypoxemia in early acute respiratory distress syndrome. AmJRespir CritCare Med.2006;174(3):268-78.

8- Merriam-Webster. Merriam-webster's medical dictionary. Springfield, Massachusetts: Merriam-Webster; 1995.

9- Behnam Moghadam M, Azimian J, Abdi N, Mirzaee MS. Non invasive hemodynamic monitoring in critical care units: Review article. J Anesthesiol Pain. 2015;5(2):72-81. [Persian]

10- Valentin A, Ferdinande P, ESICM Working Group on Quality Improvement. Recommendations on basic requirements for intensive care units: Structural and organizational aspects. Intensive Care Med. 2011;37(10):1575-87.

11- Tobin MJ. Principles and practice of mechanical ventilation. Tobin MJ, editor. 3rd edition. New York: McGraw Hill Professional; 2012.

12- McGee DC, Gould MK. Preventing complications of central venous catheterization. $\mathrm{N}$ Engl J Med. 2003;348(12):1123-33.

13- Antonelli M, Conti G, Esquinas A, Montini L, Maggiore $\mathrm{SM}$, Bello G, et al. A multiple-center survey on the use in clinical practice of noninvasive ventilation as a first-line intervention for acute respiratory distress syndrome. Crit Care Med. 2007;35(1):18-25.

14- Topp R, Ditmyer M, King K, Doherty K, Hornyak J 3rd. The effect of bed rest and potential of prehabilitation on patients in the intensive care unit. AACN Clin Issues. 2002;13(2):263-76.

15- Westerdahl E, Lindmark B, Eriksson T, Hedenstierna G, Tenling A. The immediate effects of deep breathing exercises on atelectasis and oxygenation after cardiac surgery. Scand Cardiovasc J. 2003;37(6):363-7.

16- Genc A, Koca U, Gunerli A. What are the hemodynamic and respiratory effects of passive limb exercise for mechanically ventilated patients receiving low-dose vasopressor/inotropic support?. Crit Care Nurs Q. 2014;37(2):152-8.

17- Brimioulle S, Moraine JJ, Norrenberg D, Kahn RJ. Effects of positioning and exercise on intracranial pressure in a neurosurgical intensive care unit. Phys Ther. 1997;77(12):1682-9.

18- Loram L. The Cardiopulmonary effect of passive movement. S Afr J Physiother. 2002;58(3):27-32.

19- Czell D, Schreier R, Rupp R, Eberhard S, Colombo G,
Dietz V. Influence of passive leg movements on blood circulation on the tilt table in healthy adults. J Neuroeng Rehabil. 2004;1(1):4.

20- Richard R, Staley M, Miller SF. The effect of extremity range of motion on vital signs of critically 111 patients and patients with burns: A Pilot Study. J Burn Care Res. 1994;15(3):281-4.

21- Achugbue FS. The effects of therapeutic passive movement on cardiovascular response in stroke patients. Res J Med Sci. 2009;3(1):12-5.

22- Amidei C, Sole ML. Physiological responses to passive exercise in adults receiving mechanical ventilation. Am J Crit Care. 2013;22(4):337-48.

23- Monnet X, Teboul JL. Passive leg raising. Intensive Care Med. 2008;34(4):659-63.

24- Venturelli M, Amann M, Layec G, McDaniel J, Trinity JD, Fjeldstad AS, et al. Passive leg movement-induced hyperaemia with a spinal cord lesion: Evidence of preserved vascular function. Acta Physiol (Oxf). 2014;210(2):429-39.

25- Savi A, Maia CP, Dias AS, Teixeira C. Hemodynamic and metabolic effects of passive leg movement in mechanically ventilated patients. Rev Bras Ter Intensiva. 2010;22(4):315-20.

26- McDaniel J, Fjeldstad AS, Ives S, Hayman M, Kithas P, Richardson RS. Central and peripheral contributors to skeletal muscle hyperemia: Response to passive limb movement. J Appl Physiol (1985). 2010;108(1):76-84.

27- Fukuda S, Shimada K, Kawasaki T, Kono Y, Jissho S, Taguchi H, et al. "Passive exercise" using whole body periodic acceleration: Effects on coronary microcirculation. Am Heart J. 2010;159(4):620-6.

28- Thelandersson A, Volkmann R, Cider A. Blood flow velocity and vascular resistance during passive leg exercise in the critically ill patient. Clin Physiol Funct Imaging. 2012;32(5):338-42.

29- Younis G, Sayed Ahmed S. Effectiveness of passive range of motion exercise on hemodynamic parameters and behavioral pain intensity among adult mechanically ventilated patients. IOSR J Nurs Health Sci. 2015;4(6):4759.

30- Koch SM, Fogarty S, Signorino C, Parmley L, Mehlhorn $\mathrm{U}$. Effect of passive range of motion on intracranial pressure in neurosurgical patients. J Crit Care. 1996;11(4):176-9.

31- Pires-Neto RC, Kawaguchi YMF, Hirota AS, Fu C, Tanaka C, Caruso P, et al. Very early passive cycling exercise in mechanically ventilated critically ill patients: Physiological and safety aspects--a case series. PLoS One. 2013;8(9):e74182.

32- Marino PL. Marino's The ICU Book. Alphen aan den Rijn: Wolters Kluwer Health; 2013.

33- Asgari MR, Soleimani M. Comprehensive book on intensive nursing care in ICU, CCU and dialysis wards. $3^{\text {th }}$ edition. Tehran: Boshra; 2004. [Persian] 